\title{
Equipment Maintenance Support Analysis Model Based on Field-Theory
}

\author{
Wei-yi WU, Ping GU, Jie Liu, Bin Liu \\ Department of Equipment Command \&Management \\ Ordnance Engineering College \\ Shijiazhuang 050003, China \\ weiyi1019@163.com
}

\begin{abstract}
At present military activities have been influenced deeply by the information revolution, in the same way the equipment support activities are confronted with tremendous challenge and many problems appeared. The equipment support ability is not satisfied with its target in the traditional method along with in-depth research, so needed to quest for a new research angle of view. Through the investigation of electric-particles' movement in physics field, a field-theory point of view from the comparability between the electricparticles and the equipment support elements is introduced which based on spatial distributing of equipment support elements around battlefield and characteristic of support activities. Moreover, the theory and method of the field-theory in the transfer of the support element and energy in support activity is described as the basic for the next application research. The appropriate combination between the natural science and military science has the important meaning for enrichment and development of equipment support theory.
\end{abstract}

Keywords: equipment support; field-theory; support field; support potential

\section{INTRODUCTION}

Along with the transformation from the industrialization to the informationization of the world, the information warfare has begun to take the place of mechanization warfare which becomes the latest war type in the war modality and theory, in the same way the radical reform has changed in the equipment maintenance support, which can offset the insufficient of the support resource and support activities.

Wartime equipment maintenance support force is usually formed of concomitant support force, mobile support force and immovable support facilities, through which a maintenance support system is constructed of the combination of the located support and mobile support. The located maintenance facilities usually contain the integrated maintenance instruments, which can provide the broken or stricken equipments with more complicated and comprehensive maintenance, thus these facilities play an important role in the support system. The different levels of support facilities such as support station and basement have the different maintenance ability, which the stronger one will form the high support potential energy, while the weaker one form the low potential energy. Because of the diversity, the energy stream of the technology and materials will flow from the high facilities to others with the lower potential energy, until the support requirements from combatant forces are fulfilled.

\section{DESCRIPTION OF THE EQUIPMENT MAINTENANCE SUPPORT PROCESS}

Present-day equipment maintenance support usually adapts the concept of the "Forward-Replacing and Backward Maintaining". The basic process of this concept is to open up bases backward of the operation area, in charge of providing sufficient maintenance support to the operation force. In order to reduce the back moving distance between the broken equipment and the maintenance bases and improve the support efficiency, maintenance stations could be sent out from bases to provide located maintenance and direct support in the important operation direction. Along with operation forces forward, concomitant support teams are configured to replace disabled parts of unworkable equipments with new ones. Besides, mobile maintenance teams are kept in case that concomitant support teams might be needed reinforcement. To ensure the quality and efficiency of the support service, stations and bases will reserve some of support resource such as maintenance workbench and repair parts.

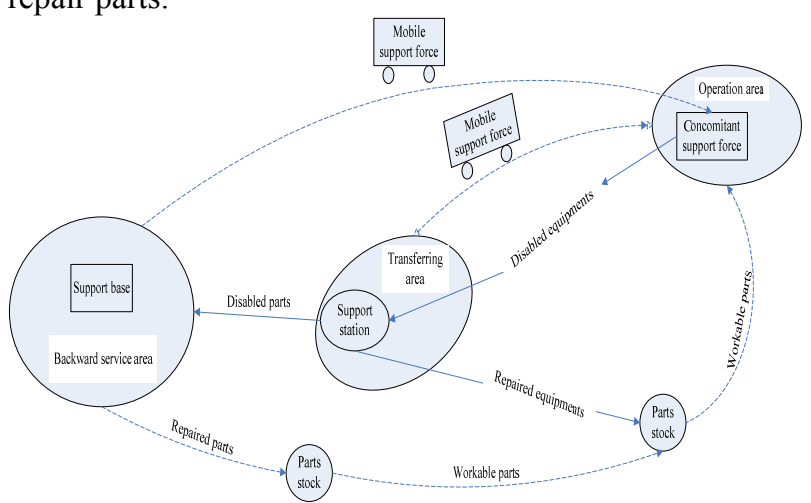

Figure 1. Sketch map of equipment maintenance support

Figure 1 depicts the maintenance support process of stricken equipments. Once stricken, an equipment will be firstly detected and disable parts will be replaced on the spot. If not successful, the equipment will secondly moved to the nearest support station and undergone further replacement. If reserved new parts are not sufficient, it will have to wait for them to arrive. The disabled part removed from the equipment will be repaired if there is idle workbench and added to stock of the station. 
But if the disabled parts can not be repaired at the station, they will be further moved back to a support base and go through the same process at the station. If there are not sufficient stock for repairing the parts at the base, they will have to wait too.

\section{EQUIPMENT SUPPORT POTENTIAL ENERGY AND SPACE DISTRIBUTION}

According to modern theory of Physics, "field" is the concept defining the space distribution of any physical measure. Equipment support is formed of activities carried out in some space area, whose elements have defined time, space distribution, inter-connection, reciprocity and interaction between each other. In other words, this is one kind of the field. Through belonging to the category of strategics, equipment support possesses essential elements to be as a field, which including the specific space area(as the operation area), the field elements(as the support units), the distributing discipline, functioning and inter-connection among the elements. So, in consideration of the comparability with Physics, equipment support field was defined as the distribution of inter-connected support elements, and distribution of the inter-impacting effect in the support process, being known as Support Field (SF).

According to the combatant intention, the support task and environment of the battlefield, equipment support forces will be organized into support groups. By depicting support power of these groups as support energy of SF, they should be scientifically and efficiently managed by theater equipment support commanding organizations. Driven by requirements, support energy will flow from support forces to its objects. Because of the diversity of support energy between support forces, the process exists which the energy flowing from a higher level force to the lower level one, it can be described as different potential energy between support forces. Based on the general potential definition, some strength drives an object moving from a high potential point to a low potential point, which meaning the space or time is concerned in the support energy management system, the strength that drives support energy flowing should has the same direction with the support potential energy guarantee.

Support units and support objects can be viewed as points in the operation area. In Physics, electric charge in vacuum will attract the electric field around, which implements the inter-connection between each other. Similarly, on defining equipment support field, the points of support units and objects can be defined as a kind of electric charge, by which the support energy of a support unit and requirement of a support object become quantity of electricity. It defines the support units to be positive and the support objects to be negative. The more quantity of electricity a support unit or object has, the higher level of support power or requirement it receives.

According to theory of Physics, the potential of electric field attracted by the electric charge is

$$
U=\frac{Q}{4 \pi \varepsilon_{0} \mathrm{r}}
$$

Where $\mathrm{Q}$ is the quantity of the electric charge, $\mathrm{r}$ is the distance between the charge and the measure point, $\varepsilon_{0}$ is the constant.

Formula (1) shows that electric potential at a point in the electric field is in direct proportion to quantity of electricity of the charge, and is inverse ratio to distance between two points. Once a support unit ws abstracted as the electric charge with specific quantity of electricity, its support potential can be calculated by formula (1). What should be illuminated is that it is a complicated process that support potential diffuses and makes weaker while the distance raises, which is not simply depicted by formula(1). Simular to the electric potential, the total support potential energy will be the superposition of all support fields attracted by the support units will be more than one is deployed.

\section{EQUIPMENT MAINTAINING SUPPORT RESOURCE DISPATCHING DIRECTION MODEL}

Under the field-theory, the final goal of equipment support activities is to achieve dynamic balance of energy within support field, in other words, Once the support requirement changes from a support object due to changing the task or the combat intensity, the energy balance state is broken. In order to regain this balance, some measurable support energy must be transferred from source of the support field to the required force, and the measurable support energy is optimal in the resource dispatching. In the other hand, in support field, potential energy of support source, requirement force and resistance in support process are denied to be function of quantity of support resource flow. Thus, according to energy transmission mechanism in support field, this function can be established recur to proper mathematic approach.

The equipment support resource dispatching model has following hypothesis: in support field, there are support source (mainly the support units), resource requirement (mainly the combat units), and one or more resource dispatching routes. The upper bound of each support source and the low bound of each requirement is specified. Figure 2 shows the space relation between elements in equipment support field.

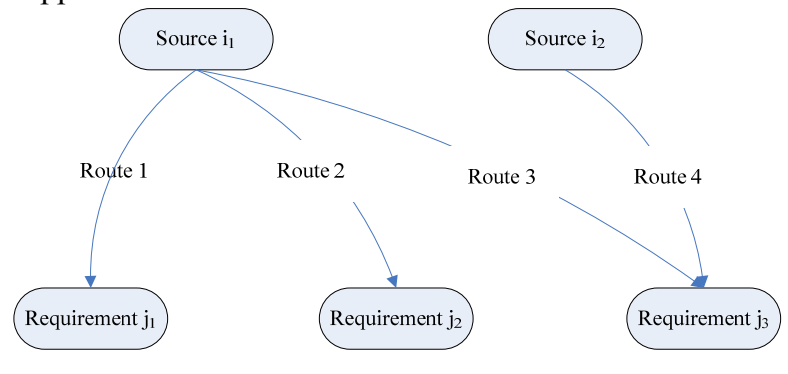


Figure 2. sketch map of space relation between support field elements

Following variables are included in equipment support resource dispatching model: $i(i=1,2, \cdots, m)$ is support source; $j(j=1,2, \cdots, n)$ is requirement; $\alpha(\alpha=1,2, \cdots, \mathrm{k})$ is resource dispatching route between source and requirement; $S_{i}$ is resource amount at source point $\mathrm{i} ; d_{j}$ is requirement amount from point $\mathrm{j} ; X_{\alpha}$ is the resource amount on route $\alpha ; U_{s i}$ is potential energy of meta amount of resource at source point $i$, which is function of $S_{i} ; U_{d j}$ is potential energy of meta amount of resource at requirement point $\mathrm{j}$, which is function of $d_{j}$; $R_{\alpha}$ is process resistance when meta amount of resource goes through route $\alpha$, which is function of $x_{\alpha} ; S_{i \max }$ is upper bound of support $i$; $d_{j \text { min }}$ is lower bound of requirement $\mathrm{j} ; \quad \alpha_{\alpha, \mathrm{i}} \in\{0,1\} \beta_{\alpha, \mathrm{j}} \in\{0,1\}$, where "1" means route $\alpha$ goes across source $i$ or requirement $j$, and "0" means it does not.

The object of this model is to maximizing the level support activities fulfilling support resource requirement, which means maximizing the margin between the total resource effect at requirement points and sumption of total resource effect at source points and resistance of all routes, as follows:

$$
\begin{array}{ll}
\max \left\{\sum_{j=1}^{n} \int_{0}^{d_{j}} U_{d j}(x) d x-\sum_{i=1}^{m} \int_{0}^{s_{i}} U_{s i}(y) d y-\sum_{\alpha=1}^{\mathrm{k}} \int_{0}^{\mathrm{x}_{a}} R_{\alpha}(z) d z\right\} \\
\text { s.t. } \quad \sum_{\alpha=1}^{\mathrm{k}} \alpha_{\alpha, \mathrm{i}} X_{\alpha}=s_{i} \leq s_{i \max }, \forall i \\
& \sum_{\alpha=1}^{k} \beta_{\alpha, \mathrm{j}} X_{\alpha}=d_{j} \geq d_{j \min }, \forall j \\
& E_{\alpha}=U_{d j}-U_{s i}-R_{\alpha}>0, \forall \alpha, \mathrm{i}, \mathrm{j} \\
& \alpha_{\alpha, \mathrm{i}}, \beta_{\alpha, \mathrm{j}} \in\{0,1\} \\
& d_{j}, S_{i}, U_{d j}, U_{s i}, x_{\alpha} \geq 0
\end{array}
$$

Where formula (2) is the object function, which shows mechanism of support resource flow within support field. Formula (3) and (4) give restriction to resource dispatching, which means the total resource amount goes through all routes, should not overrun upper bound of support source. And to any requirement, that amount should not be fewer than lower bound of the requirement. Formula (5) gives the work condition for the whole support system, which means the support field intensity around dispatching routes is hyper-zero. The differential coefficient in the object function means the potential energy of the support field and process resistance will continually change along with the change of the support resource dispatching amount.

\section{CONCLUSION}

Equipment maintenance support is a complicated subject under condition of informationization, where both the maintaining support process and resource dispatching have to be comprehensive considered. In order to study the subject more precisely, the paper put forward a field theory by analogy with Physical field to reveal the self-adaptive quality of equipment maintenance support, which provides analyzing and modeling methodology for self-adaptive equipment support research.

\section{REFERENCES}

[1] Guvenc Sahin, Haldun Sural. A review of hierarchical facility location models[J]. Computers \& Operations Research, 2007, 34:2310-2331.

[2] Marianov V, Serra D. Probabilistic maximal covering locationallocation for congested system[J]. Journal of Regional Science, 1998,38:401-424.

[3] Marianov V, Serra D. Location-allocation of multiple-server service centers with constrained queues or waiting times[J]. Annals of Operations Research,2002,111:35-50.

[4] Marianov V, Serra D. Hierarchical location-allocation models for congested systems[J]. European Journal of Operational Research, 2001,135:195-208.

[5] van JCW, Ommeren, Bumb A F, Sleptchenko A V. Locating repair shops in a stochastic environment[J]. Computers \& Operations Research, 2006,33:1575-1594.

[6] Avsar Z M, Zijm W H M. Resource-constrained two-echelon inventory models for repairable item systems[R/OL]. External research report [2007-08-23]. http: // purl.org/utwente/30579.

[7] Marvin A A Jr, Sukran N K, Basheer M K. An empirical comparison of Tabu search, simulated annealing, and genetic algorithms for facilities location problems[J]. Int J Production Economics,2006,103:742-754.

[8] Bin song, Chuan xin Gong, Zhao Fu meng. An New Logic of Organization Reform of Equipment Support in Information Condition[J]. JOURNAL OF ACADEMY OF ARMORED FORCE ENGINEERING. 2006,20:324-327.

[9] Song wei Bai, Chuan xin Gong, Pu Gu. Research on Innovation of Training Content System of Equipment Support for the Integrated Joint Operations[J]. JOURNAL OF ACADEMY OF ARMORED FORCE ENGINEERING. 2006,20:763-767.

[10] Yun jie Liu, Chuan xin Gong, Wen Zhou. Research on application of model-driven architecture in information system[J]. COMPUTER ENGINEERING AND DESIGN. 2006,27:1021-1025.

[11] Li min Feng, Chuan xin Gong, Xiao li Gao. Analysis of information flow in equipment support chain[J]. JOURNAL OF NAVAL AERONAUTICAL ENGINEERING INSTITUTE. 2010,22:113-114.

[12] Liang hua Xia, Ping Gu. Research of Flexible Assignment for Equipment Support Resources[J]. JOURNAL OF THE ACADEMY OF EQUIPMENT COMMAND \& TECHNOLOGY. 2004,15:241245. 